\title{
Microfluidics-Based Reactors for Safe Fluorinations Using Elemental Fluorine
}

\author{
Michael D. Turnbull ${ }^{\mathrm{a} *}$, Neil B. Carter ${ }^{\mathrm{a}}$, Stephen Dennison ${ }^{\mathrm{b}}$, Julie Deacon ${ }^{\mathrm{b}}$, and Richard Holley ${ }^{\mathrm{b}}$
}

\begin{abstract}
The design, development and initial operation of a new type of fluorine microreactor are described. Its use in agrochemical discovery chemistry is illustrated with simple examples of electrophilic fluorination.
\end{abstract}

Keywords: Fluorination · Fluorine gas · Microfluidics · Microreactor

\section{Introduction}

Shortly after his isolation of elemental fluorine in 1886, Moissan reported that reactions between fluorine and organic compounds were 'spontaneous and catastrophic', producing clouds of soot and HF. Successful direct fluorination began only from the 1930s onwards when it became apparent that success demanded efficient dissipation of the heat of reaction (a typical exotherm being $450-500 \mathrm{~kJ} \mathrm{~mol}^{-1}$, ignoring the heat of solution of the produced HF) [1]. An attractive method for conducting such reactions, allowing good control of exothermicity, maintaining good stoichiometry control and with small fluorine inventories is provided by microfluidic reactor systems [2]. Among these are the reactor systems developed by the groups of Jensen [3] and Chambers [4].

The reactors developed by Jensen's group at MIT are fabricated using silicon chip micro-machining techniques developed for the electronics industry [3]. A silicon wafer is subjected to photolithography,

${ }^{*}$ Correspondence: Dr M.D. Turnbulla

Tel.: +441344 414789

Fax: +44 1344455629

E-Mail: mike.turnbull@syngenta.com

asyngenta

Lead Finding Chemistry

Jealott's Hill International Research Centre

Bracknell, Berkshire

RG42 6EY, UK

${ }^{\mathrm{b}} \mathrm{CRL}$ Ltd

Dawley Road

Hayes, Middlesex

UB3 $1 \mathrm{HH}$, UK etched with $\mathrm{KOH}$ to form channels which are then coated with a layer of silicon oxide which has a thin film of nickel deposited over the surface to protect the silicon from $\mathrm{HF}$ etching. Fluorine in concentrations of $0-25 \% \mathrm{v} / \mathrm{v}$ is then introduced and the substrate and solvent are pumped via syringe pump into the reactor channel. The latest reactors of this type are suitable for direct electrophilic fluorination of simple organic molecules such as toluene [3].

The design of Chambers and co-workers is based on a single nickel block milled out to form a microchannel and capped with a plate. Gas and substrate solution flow into the channel and reaction occurs [4]. Products are then trapped in a fluoroethylene polymer trap and waste gases go to a scrubber. This device has been shown to be suitable for a diverse range of organic chemistries involving fluorine, including $\beta$ dicarbonyl fluorination, $\mathrm{SF}_{5}$ formation, electrophilic fluorination of aromatics, polyfluorination [4] as well as fluorinecatalysed oxidations and Baeyer-Villiger reactions [5].

Fluorinated aromatics such as Tefluthrin (Fig. 1) are a very common motif in agrochemicals due to the properties fluorine imparts upon biologically active molecules [6]. Tefluthrin is a pyrethroid insecticide active ingredient, which is effective in the control of lepidoptera, coleoptera and diptera on wheat, maize (corn) and other crops [7].

Direct fluorination is therefore a very attractive process for discovery chemistry in agrochemicals research. It has been our aim to build on the ideas and knowledge of direct fluorination with microreactors available in the literature, to develop a practical reactor system for routine use by synthetic chemists within Syngenta at scales suitable for discovery chemistry. A collab-

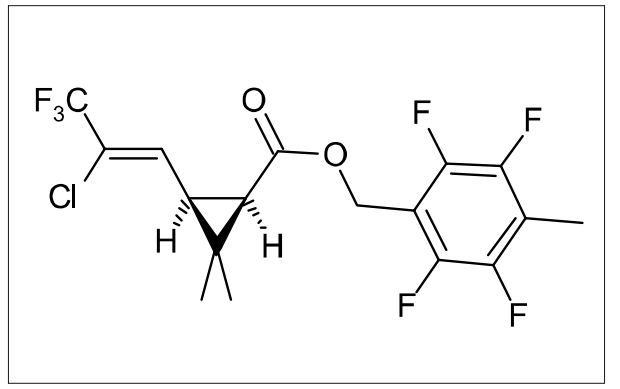

Fig. 1. Tefluthrin, a pyrethroid insecticide

oration between Syngenta and CRL, a specialist in micro-engineered devices, has been developed to the point where a second generation reactor has been produced and is in operation at the Syngenta Jealotts Hill research laboratories, UK. The evolution, design and operation of this reactor system are presented below.

\section{Reactor Design}

The design concept for our reactor was inspired mainly by the designs of Chambers and the general organic synthesis reactors produced by Haswell and coworkers [8]. The brief was for a system which was safe to use, capable of undertaking the fluorination of various organic substrates, easy to operate and quickly stripped down, cleaned and reassembled. From this, the concept of a 'sandwich' reactor was developed in which a high grade, stainless steel body would hold three plates of PTFE, one of which would feature the gas and substrate inlets and a product/waste gases outlet. A second PTFE plate would have the channel cut into it and a third plate would then cap the channel. Pressure would be applied via a larger, screw-driven metal top-plate to 


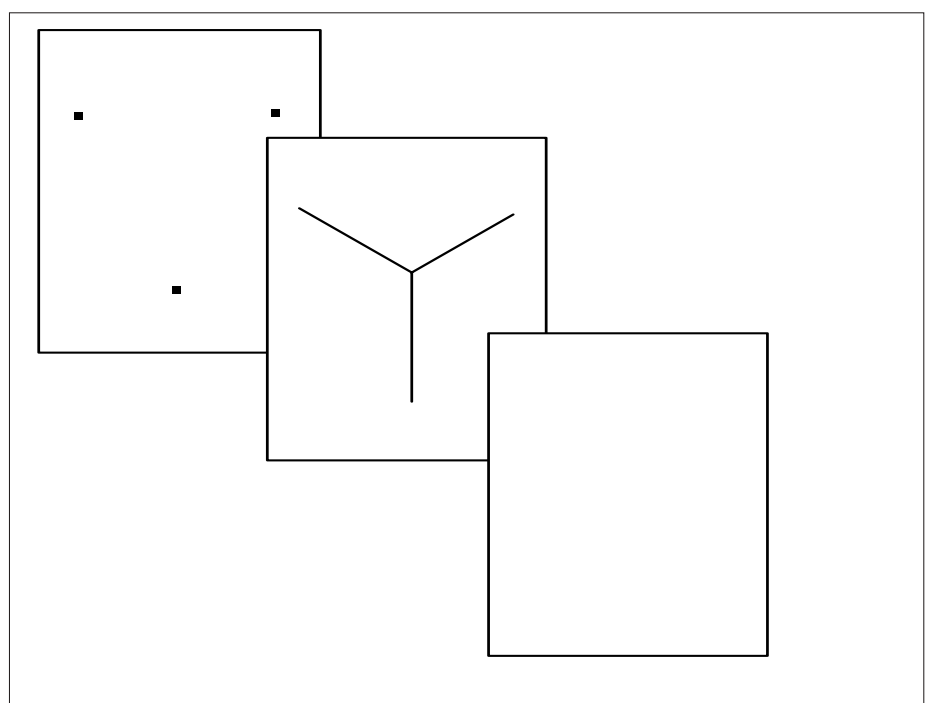

Fig. 2. An exploded view of the reactor core sandwich

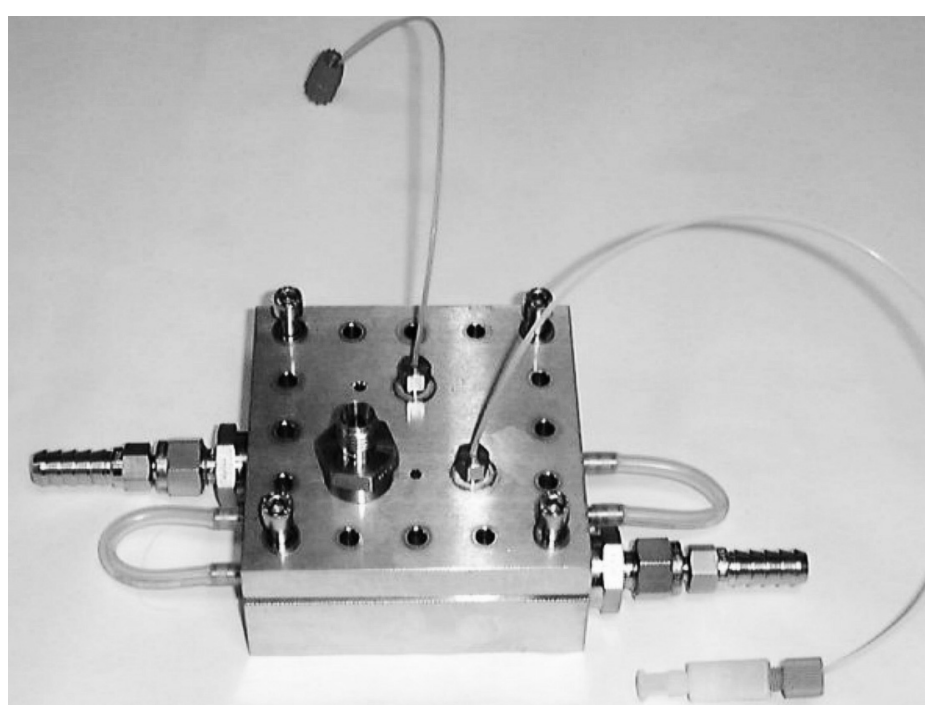

Fig. 3. The prototype reactor developed to test the principles of our new design

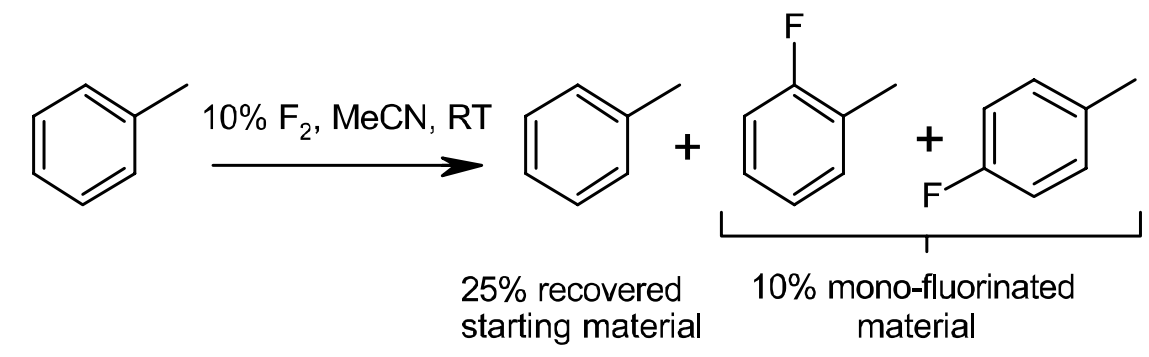

Scheme 1. Initial attempts to fluorinate toluene with fluorine gas inside the prototype reactor

compress the plates and make the reactor 'core' gas tight. An exploded illustration of a typical gasket assembly is illustrated in Fig. 2.

\section{Development of a Prototype Reactor}

To test the feasibility of this approach, a prototype was constructed and tested for some simple fluorination substrates. In this first reactor (Fig. 3) a solid steel block was milled out to hold the 'sandwich' core and covered with a flat 'top plate'. Steel bolts were then used to secure the unit and make it gas tight. Input gases were controlled via two mass flow controllers (Hastings) and products and waste gases were passed into a PTFE product collection vessel and the waste gases passed to a scrubber unit charged with $20 \%$ w/v KI solution. Channels throughout the base of the steel body were designed to allow cooling fluid from a heat exchanger to pass through and provide modest temperature control.
There are some important advantages over existing designs with this type of construction. Firstly, the channel in which reaction occurs can be altered, simply by changing the PTFE 'channel gasket' in the core, thus increasing or decreasing reaction time without altering flow rates. Secondly, the effect of differing construction materials can be investigated by inserting thin foils of different material between the PTFE gasket plates; for example a nickel walled reactor can be constructed by inserting nickel foils between the PTFE surfaces. Thirdly, any defects, deformations, decomposition or blockages in the reactor can be easily dealt with simply by changing the cheaply produced PTFE reactor core. These features give added flexibility and ease of use to the design.

Fluorination using our prototype reactor was initially attempted using toluene as the substrate and acetonitrile as solvent, with a $10 \% \quad \mathrm{~F}_{2}$ concentration. Solvent/reagent flows of $1 \mathrm{ml} \mathrm{h}^{-1}$ and gas flows of $10 \mathrm{ml}$ $\mathrm{min}^{-1}$ were employed (Scheme 1). Under these conditions it proved possible to obtain fluorine-enriched materials, which were analysed by GC-MS and tentatively assigned as a mixture of 2-fluorotoluene and 4 -fluorotoluene ( $10 \%$ yield) with toluene itself $(25 \%)$. The discrepancy in mass-balance was assumed to arise from poly-fluorinated materials, forming tar and volatile materials evaporating in the gas stream. Recovery experiments performed later showed that at the typical gas flows used, up to $c a$. $10 \%$ of relatively volatile product mixture could be lost through evaporation during passage through the reactor.

This result along with other experiments conducted using the prototype were felt promising enough to encourage us to develop a second-generation microreactor more suited to routine use, allowing the development of more applicable chemistries.

\section{Second-Generation Microreactor}

Our design for the new microreactor closely followed the concepts of the old design, but with improvements to make the reactor more reliable and easier to use. Instead of using bolts to clamp the gaskets together, the new design has a hinge between the top-plate and the base with a bar to secure the plates together and a foot mounted on a screw-press to provide the required pressure to hold the gaskets together in a gas-tight seal. The reactor has also been designed to accept a 'notched' set of gasket plates, so accidental incorrect insertion becomes more difficult. The channel gasket for the new reactor is illustrated in Fig. 4 and Fig. 5.

The reactor was also designed to reduce the time that the liquid and gaseous reagents were in contact after emerging from the microfluidic channel. The new re- 


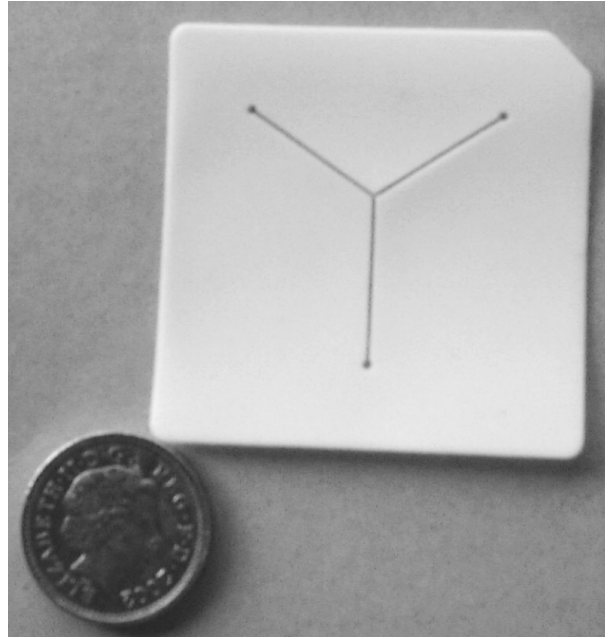

Fig. 4. The new 'channel gasket' (placed next to a one pound coin for size comparison), note the 'notched' design to assist in gasket orientation

actor was furnished with a product trap mounted on the base of the reactor body, so that it can be immersed in a dry ice/acetone trap. At $-78{ }^{\circ} \mathrm{C}$, most common solvents used in fluorination reactions will solidify, thus reducing the reaction/absorption of waste gases. The gas/liquid mixture emerging from the channel enters a wide-bore tube ( $2.0 \mathrm{~mm}$ diameter), in which the waste gases are de-trained from the liquid, thus reducing any post-channel reaction. Inside the product trap, the liquid product drops to the base of the PTFE vessel, where it is frozen, and the gas flows out to the waste gas handling system. As in the prototype reactor, the reaction temperature is moderated by pumping heating/cooling fluids through the body of the reactor and solvent/reagent inputs are handled via a syringe pump (Fig. 6).

The reactor has proven suitable for fluorinations in a range of solvents including acetonitrile, $\mathrm{HCO}_{2} \mathrm{H}$, conc. $\mathrm{H}_{2} \mathrm{SO}_{4}$ and $\mathrm{MeOH}$. It has been possible to run reactions at temperatures from $-30{ }^{\circ} \mathrm{C}$ to $+40{ }^{\circ} \mathrm{C}$ (with judicious solvent choice). To date along with simple aromatic electrophilic substitutions it has also been used to fluorinate $\beta$-diketones.

Our initial studies with the second-generation reactor focused on the transformation of anisole to fluoroanisole and nitrobenzene to fluoronitrobenzene. It was thought that the electron-rich anisole and the electron-poor nitrobenzene would serve as two likely extreme substrates for electrophilic fluorination, with other molecules falling between these two in terms of reactivity. Fluorination of toluene was also studied, as it would allow a more direct comparison between the performance of our system and those of Chambers [4] and Jensen [3].

\section{Fluorination of Anisole, Nitro- benzene and Toluene}

For this study anisole (1), nitrobenzene (2) and toluene (3) were passed through the reactor as solutions in acetonitrile $(100 \mathrm{mg}$ $\mathrm{ml}^{-1}$ ) at a rate of $1.0 \mathrm{ml} \mathrm{h}^{-1}$, over a period of $60 \mathrm{~min}$ and with a gas flow $\left(10 \% \mathrm{~F}_{2}\right)$ of $10 \mathrm{ml} \mathrm{min}^{-1}$ to accomplish the fluorination (Scheme 2). Analysis of the resulting products by GC (against authentic samples of the fluoroaromatics formed) showed that all three substrates underwent mono-fluorination to some extent. As expected, the best yields were obtained with the electron-rich anisole and the poorest arose from fluorination of nitrobenzene (see Table).

The expected, preferred regio-isomers arising from electrophilic substitution onto the aromatic ring predominate; in the case of anisole none of the disfavoured 1c was detected in the reaction showing the strong effect of the activation afforded to the ortho and para positions by the electron-donating methoxy group. This trend is also evident in the case of toluene although the effect is not as pronounced $(1 \% \mathbf{3 c}$ detected), in line with the weaker electron-donat- ing characteristics of the methyl group. Jensen and co-workers [3] undertook experiments under similar conditions and obtained similar combined yields of $10-14 \%$ in acetonitrile, with equal or lower selectivities obtained.

More detailed studies investigating the effects of variation of temperature, solvent and $F_{2}$ concentrations have been undertaken with these substrates and these results will be reported elsewhere in due course.

\section{Fluorination of $\beta$-Diketones}

For our studies 2,2-6,6-tetramethyl-3,5heptanedione (4) was passed through the reactor as a solution in either acetonitrile or $\mathrm{HCO}_{2} \mathrm{H}\left(50 \mathrm{mg} \mathrm{ml}^{-1}\right)$ at a rate of $0.5 \mathrm{ml} \mathrm{h}^{-1}$, over a period of 30 min and with a gas flow $\left(10 \% \mathrm{~F}_{2}\right)$ of $10 \mathrm{ml} \mathrm{min}^{-1}$ to accomplish the fluorination. Analysis of the resulting products showed conversion to 4-fluoro-2,26,6-tetramethyl-3,5-heptanedione (5) with no di-fluorination detected in either case (Scheme 3).

The yields in acetonitrile and $\mathrm{HCO}_{2} \mathrm{H}$ were $68 \%$ and $86 \%$ respectively, with good

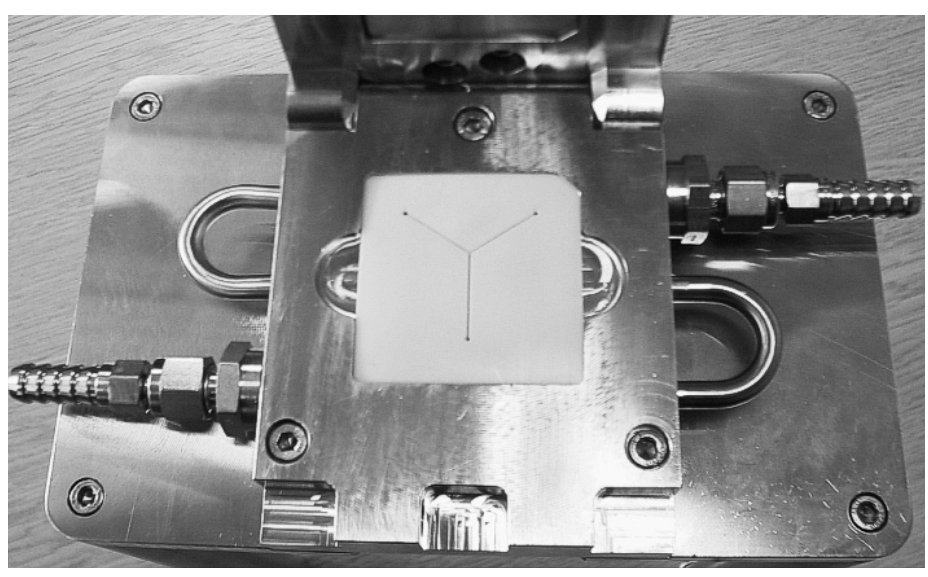

Fig. 5. A top down view of the reactor channel gasket as fitted into the reactor.

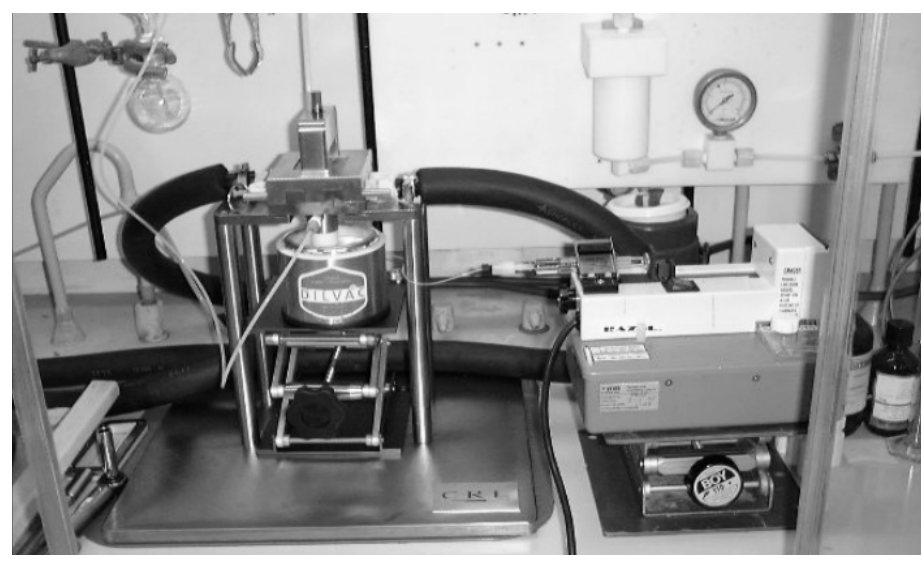

Fig. 6. The second generation reactor installed and in operation in our laboratories. 


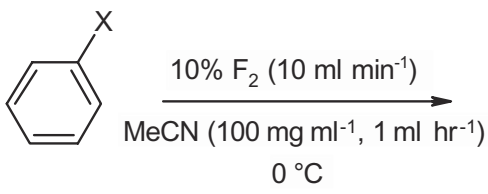

$1, \mathrm{X}=\mathrm{OMe}$

2, $\mathrm{X}=\mathrm{NO}_{2}$

$3, \mathrm{X}=\mathrm{Me}$
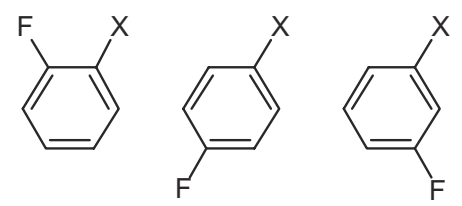

b

$c$
Scheme 2. Fluorination of anisole (1), nitrobenzene (2), and toluene (3), with elemental fluorine<smiles>CC(C)(C)C(=O)/C=C(\O)C(C)(C)C</smiles>

4<smiles>CC(C)(C)C(=O)/C=C(\O)C(C)(C)C</smiles>

4

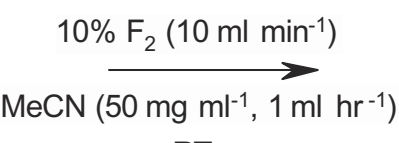

RT

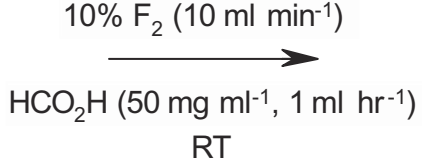

RT<smiles>CC(C)(C)C(=O)C(F)C(=O)C(C)(C)C</smiles>

5

$68 \%$ Yield<smiles>CC(C)(C)C(=O)C(F)C(=O)C(C)(C)C</smiles>

5<smiles>CC(C)(C)C(=O)/C=C(\O)C(C)(C)C</smiles>

8\% Recovery<smiles>CC(C)(C)C(=O)/C=C(\O)C(C)(C)C</smiles>

8\% Recovery

Scheme 3. Fluorination of $\mathbf{4}$ with elemental fluorine

conversion of starting materials $(76 \%$ and 94\% respectively) and only small amounts of starting material recovered in each case. Perhaps most pleasing was the absence of significant side products indicating a clean conversion to product. The superior yield with $\mathrm{HCO}_{2} \mathrm{H}$ as solvent is attributed to the higher concentration of the enol form of the diketone in acidic solvent, which it is assumed, is the species through which reaction occurs.

Fluorination of $\mathbf{4}$ has been performed previously by Purrington and co-workers [9] using the silyl enol ether of $\mathbf{4}$ and fluorination with $5 \% \mathrm{~F}_{2}$ in $\mathrm{CFCl}_{3}$ at $-78^{\circ} \mathrm{C}$. The reported yield for this transformation was $40 \%$ so these results represent a significant advance.

\section{Conclusions}

The work discussed herein illustrates the design, construction and performance of a microfluidic fluorine reactor. It is hoped this report illustrates the special importance placed on fluorination in agrochemical discovery chemistry and the possibilities of direct elemental fluorination as a tool, which with the correct apparatus can be utilised in routine discovery chemistry, safely and at a suitable scale.

\section{Acknowledgments:}

We wish to thank all our colleagues at Syngenta and CRL for the advice, support, and technical help we have received throughout this project.

Received: January 12, 2004

Table. Yields of mono-fluorination products from the fluorination of anisole, nitrobenzene and anisole

$\begin{array}{lccccc} & \text { Recovered }(X) & \text { Yield Xa } & \text { Yield Xb } & \text { Yield Xc } & \text { Combined Yield } \\ \text { Substrate }(X) & \% & \% & \% & \% & \% \\ \text { Anisole (1) } & 8 & 61 & 27 & 0 & 88 \\ \text { Nitrobenzene (2) } & 75 & 1 & 1 & 5 & 7 \\ \text { Toluene (3) } & 57 & 8 & 7 & 1 & 16\end{array}$

[1] J. Hutchinson, G. Sandford, in 'Topp. Curr. Chem.', Vol 183, Ed. R.D. Chambers, Springer-Verlag, Berlin, 1997.

[2] For a general review of microfluidic reactor technology see: P.D.I. Fletcher, S.J. Haswell, E. Pombo-Villar, B.H. Warrington, P. Watts, S.Y.F. Wong, X. Zhang, Tetrahedron 2002, 58, 4735-4754.

[3] N. de Mas, A. Günther, M.A. Schmidt, K. F. Jensen, Ind. Eng. Chem. Res. 2003, 42 , 698-710.

[4] (a) R.D. Chambers, R.C.H. Spink, J. Chem. Soc. Chem. Commun. 1999, 883; (b) R.D. Chambers, D. Holling, R.C.H. Spink, G. Sandford, Lab on a Chip 2001, 1, 132-137.

[5] R.D. Chambers, D. Holling, A.J. Rees, G. Sandford, J. Fluorine Chem. 2003, 119, 81-82.

[6] J.T. Walsh, E. Eswarkrishnan, 'Fluorine in Bioorganic Chemistry', Wiley and Sons, New York, 1991.

[7] C.D.S. Tomlin (Ed.), 'The Pesticide Manual', 13th Ed., 2003, p 934.

[8] C. Wiles, P. Watts, S.J. Haswell, E. Pombo-Villar, J. Chem. Soc. Chem. Commun. 2002, 1034-1035.

[9] S.T. Purrington, C.L. Bumgardner, N.V. Lazaridis, P. Singh, J. Org. Chem. 1987, 52, 4307-4310. 